\title{
ENTRE PROSTITUTION ET SILENCE, LA FLEUR EMPOISONNEE DE LA MODERNITE : LA FILLE ELISA (1877) D'EDMOND DE GONCOURT
}

\section{Barbara Giraud}

La Fille Elisa (1877) est le premier roman écrit par Edmond de Goncourt après la mort de son frère Jules en 1870 , alors qu'ils en avaient commencé ensemble la recherche documentaire et l'élaboration dès 1862. C'est en octobre de cette même année qu'ils étaient allés visiter la prison de femmes à Clermont d'Oise. Cette visite est relatée dans leur Journal et l'impression ressentie par les deux frères dévoile en partie leur opinion sur l'institution pénitentiaire de leur époque :

De temps en temps, quand on va au fond de la société et, pour ainsi dire, sous son théâtre, on y trouve de ces seconds dessous, machinés par la justice sociale, plus horrible que des abîmes, inconnus, ignorés, sans voix, plein d'êtres et de supplices muets - enterrement de vivants qui ne font pas un pli au tapis sur lequel les heureux vivent et dansent. (Goncourt, I : 869)

Avec ce roman, Edmond a eu « l'ambition d'écrire sur la folie pénitentiaire [et de] parler au cœur et à l'émotion [des] législateurs » ainsi qu'il l'expose dans la préface de l'édition de 1877, pour y dénoncer les pratiques carcérales de l'époque, dont celle très en vogue du système d'Auburn qui condamne les prisonnières au silence absolu. Il veut faire de La Fille Elisa un 'roman à thèse' qui explore les effets destructeurs d'une telle méthode d'incarcération sur l'être alors déshumanisé, provoquant ainsi, c'est ce dont Edmond est convaincu, la folie.

Cette « voix », celle d'Edmond seule, se fait sentir ainsi dès ce premier roman écrit sans Jules. Située à l'intersection des champs juridique et médical dans la deuxième moitié du $19^{\mathrm{e}}$ siècle, cet article est éclairé par l'analyse que Foucault expose dans Surveiller et Punir (1975). Le philosophe y évoque en effet les premiers procès qui ont accordé une valeur à la représentation médicale scientifique et plus particulièrement psychiatrique dans leur sanction juridique. C'est un véritable glissement qui s'est effectué en matière d'application de la justice à ce moment précis de l'histoire puisque selon Foucault, « un savoir, des techniques et un discours scientifique s'entrelacent avec la pratique du pouvoir de punir» pour «faire une histoire des châtiments sur fond d'une histoire des corps » (Foucault 24). Ruth Harris a montré que c'est aussi le désir de fournir des explications scientifiques aux problèmes sociaux qui a poussé la science et plus particulièrement la psychologie, à exprimer quelques unes des plus grandes anxiétés et aspirations de cette époque en donnant une perspective scientifique aux pratiques morales (Harris 18). C'est la matière même du roman d'Edmond de Goncourt. Au-delà des intentions premières de l'auteur, il s'agit de démontrer quelles sont les différentes formes de pouvoirs en jeux dans ce roman qui pose malgré tout les questions sur l'intégration des «fous » et plus particulièrement ici, des «folles" dans le corps social. Cet article s'intéressera donc aux conditions du processus de complémentarité engagé entre l'institution médicale et l'institution judiciaire au milieu du $19^{\mathrm{e}}$ siècle, pour se concentrer ensuite au « cas médical » que représente Elisa afin de mettre en évidence les discours subversifs reliés aux différentes formes de «pouvoir » dans le roman.

Le roman est composé de deux parties : la première est en fait une monographie d'une enfant de la rue. Fille d'une «faiseuse d'anges » qui grandit dans la misère et la promiscuité, elle devient prostituée à la fois par paresse car «il n'y avait en effet chez Elisa, ni ardeur lubrique, ni appétit de débauche, ni effervescence des sens » (FE 33) et parce " qu'elle se voyait délivrée de sa mère » (FE 34). Elisa se retrouve dans une maison close où ses talents et son aura de parisienne font merveille. Tombant alors dans une paresse voluptueuse, elle se distrait dans la bibliothèque de l'endroit qui regorge de romans. Or, «sur [sa] cervelle d'ignorance » (FE 55) de femme du peuple, « le roman possède une action magique. Il s'empare de la pensée de la liseuse devenue tout de suite, niaisement la dupe de l'absurde fiction. Il la remplit, l'émotionne, l'enfièvre » (FE 55). L'imagination d'Elisa devient alors la proie d'une fabulation sur l'héroïsme, l'abnégation, la chasteté, l'amour avec toujours, «à la fin, le couronnement légal des feux de l'amant devant un maire de Sparte ou d'Argos » (FE 58). Ceci, jusqu'à ce que la lecture «[soit] devenue une fureur, une rage chez Elisa ( $F E$ 59). Une tension se créé ainsi entre les désirs et les espérances de l'héroïne "que son métier lui faisait profaner à toute heure ». Son imagination ainsi ébranlée, elle se laisse enrôler un soir par un commis-voyageur "en chair et en os, le héros évoqué par [ses] rêves » ( $F E$ 64) pour lequel elle quitte la maison close. Maltraitée et dépouillée, elle entend expier ses fautes par le martyre en même temps qu'elle développe une haine «contre l'autre sexe » (FE 69). 
Redevenue prostituée, elle tombe néanmoins amoureuse d'un militaire qu'elle « se mit à aimer avec passion [...] dans l'élancement pur d'un rêve chaste » (FE 120). Le chapitre XXXIV nous informe, en creux, qu'Elisa a tué son amant ce jour-là mais ce n'est que dans la deuxième partie, aux chapitres XLVI, XLVII et XLVIII lorsque, privée de parole, les souvenirs lui remontent à l'esprit, que nous apprenons les faits: Elisa a tué par passion mais aussi pour ne pas être violéei. La deuxième partie est consacrée à la vie dans la prison après qu'Elisa a tué son amant et à sa lente déchéance physiologique et psychologique. Le roman est construit sur fond de misère populaire et de prostitution ; cependant, le but de l'auteur n'était pas de planter une autre Gervaise, cela aurait été poser la question sociale dans son entier. «Nous n’écrivons pas sur l'amélioration des classes pauvres. L'homme qui s'intéresse à la misère et aux misérables et qui garde 100000 livres de rente [...] est un farceur » (I : 929). Edmond se préoccupe uniquement de stigmatiser ce système carcéral pire que « le fouet sur les épaules nues de la femme », qui outrepasse «la peine édictée par les magistrats [en] tuant pour toujours la raison» (Préface). Le régime "d'amendement par le silence» mis au point par le système Auburnii associe dans un silence continu le travail collectif le jour dans les ateliers et l'isolement en cellule la nuit. Ce système est le triomphe d'une prison strictement corrective et normative qui impose le silence continu et érige le travail en peine en vue de modifier l'individu criminel. La minutie de l'emploi du temps fixe à la minute près chaque tâche, geste et pause dont la répétition, jour après jour enferme un peu plus la prisonnière dans un cercle de destruction psychologique. La scansion temporelle « A cinq heures et demie... A six heures et demie... A neuf heures... A neuf heures et demie... A dix heures... A quatre heures... » (FE 140) rythme ses journées, mois après mois.

L'acte criminel est, selon le monde médical de cette période, un signe de dégénération biologique et parce que la femme est naturellement passive et biologiquement déterminée, sa criminalité est immédiatement placée du côté de l'anormalité physiologique et plus particulièrement, du côté de l'incapacité mentale, de la perversion morale, de la folie. Ce discours, largement repris par les études criminologiques et sociologiques du $19^{\mathrm{e}}$ siècle (notamment par les docteurs Virey, Briquet, Tardieu, Voisin et Lombroso) place donc le sexe et ses excès comme principales causes de violence criminelle, faisant de la prostituée la figure emblématique de cette déviance. Julia Walkowitz en explique le processus dans son chapitre sur les «Sexualités Dangereuses » dans l'ouvrage de Geneviève Fraisse et Michelle Perrot, Histoire des femmes en Occident (2002). La prostituée et la prostitution sont l'enjeu de véritables débats gouvernementaux sur l'intervention de l'Etat dans la vie des pauvres : «les policiers [...] affirmaient que les maisons closes non surveillées devenaient le refuge de la criminalité et du désordre social » (Walkowitz, 449). Progressivement, et en cela Edmond de Goncourt touche au cœur du problème social qu'est le traitement des prostituées par la société, et comme Foucault l'a ensuite mis en évidence : au cœur de ce dispositif social se situe le corps et plus particulièrement ici le corps de la femme. La société lui dénie une sexualité autonome en la marginalisant, en la rendant maladive. La preuve inéluctable, selon le discours médical ambiant, de la dégénérescence de la femme criminelle est son manque d'instinct maternel (Smart 21), et la prostituée est par cela une menace directe à l'institution de la famille. Ainsi érigée en maladie mentale, la non conformité féminine justifie alors pleinement tout traitement « social» sur sa personne. La représentation de la folie permet à l'auteur de dénoncer un système qui, de la psychiatrie naissante à la façon dont la société gère collectivement la folie, fait de cette dernière une subversion de l'ordre social.

La loi de 1838 fait se rencontrer les institutions médicale et judiciaire puisqu'elle oblige les préfets et magistrats à visiter personnellement les asiles et ordonne aux médecins de consigner leur avis sur les registres. Considérée comme une œuvre «pure dans l'intention qui l'a inspirée, bonne dans son principe, sage dans ses dispositions » (Ambroise Tardieu introduction x), cette loi met en place la compétence directe et absolue des médecins dans l'appréciation de la raison ou de la folie. La médecine recherche et poursuit les désordres de l'organisme causés par les dérangements physiologiques dont celui-ci est victime. Selon A. Tardieu, alors Président de l'Académie Française de Médecine, Doyen de la Faculté de Médicine et Professeur de Médecine Légale à l'Université de Paris, les seuls progrès réels à signaler dans l'histoire de la médecine mentale sont exclusivement dus à l'application persévérante des méthodes médicales dans l'étude de la folie, c'est-à-dire à l'observation des formes, des variétés et de la marche de la maladie. Cette loi est le véritable critérium et la seule sauvegarde dans l'appréciation médico-légale de la folie (Tardieu introduction xi).

Les conditions dans lesquelles le médecin légiste intervient se situent sur trois niveaux, premièrement, dans les mesures à prendre en ce qui concerne l'isolement et le traitement, ensuite dans l'appréciation des capacités « civiles » de l'individu et enfin dans l'évaluation de la responsabilité criminelle. Tardieu évoque néanmoins un problème de vocabulaire puisque d'après lui, les termes d' « imbécilité », de « fureur », de « démence » utilisés dans le texte de loi, manquent de précision et peuvent, éventuellement mener à des abus. Cependant, puisqu'Elisa, prostituée que les lectures ont nourri de "pensées amoureuses », a tué son amant par amour, il est possible d'aborder ici son jugement par le biais de la typologie du crime passionnel, terme qui implique en luimême une «perturbation mentale ». En donnant une dimension sociale aux actes de crime passionnel, les aliénistes de l'époque en ont fait le symptôme type de la dégénérescence nationale et de l'effondrement de 
l'ordre social (Harris 291). A ce titre, le criminel passionnel bénéficiait de circonstances atténuantes puisqu'il avait obéi à des pulsions physiologiques temporaires - il échappait ainsi à la peine capitale - mais son crime justifiait un enfermement plus ou moins long en institution pénitentiaire dans laquelle il pourrait expier ses fautes. L'article 64 du Code Pénal (1810) stipule qu': «Il n'y a ni crime ni délit lorsque le prévenu était en état de démenceiii au moment de l'action ou qu'il a été contraint par une force à laquelle il n'a pu résister ». Il est aisé de le deviner, cet article a été appliqué pour le cas d'Elisa, «aux impulsions mystérieuses et secrètes auxquelles elle avait obéi » (FE 171) puisque le roman s'ouvre sur sa condamnation à mort. Le lecteur apprend que sa peine est en fait transformée en un emprisonnement à perpétuité. Il ne sait rien des raisons pour lesquelles le jugement a été remis en cause mais nous pouvons supposer qu'Elisa a été rejugée sur les bases de l'article sur le Crime Passionnel puisqu'elle a été reconnue «démente » au moment des faits. Le médecin de la prison suggère qu'Elisa, sans être folle, manque de jugement et de libre arbitre (FE 200), elle est donc reconnue irresponsable de ses actes et sujette à d'incontrôlables forces. La démence est présentée comme cette "force irrésistible» de nature physique qui entraîne l'individu dont la volonté est éteinte ou pervertie et c'est au «médecin de reconnaître et d'apprécier la réalité de cette influence » (Tardieu 45). Edmond s'est concentré dans ce roman, sur les causes de la folie : «certaines passions, vices, l'ivrognerie, la débauche peuvent à la longue amener une perversion morale et intellectuelle : ce sont des causes de la folie, ce n'est pas la folie. La distinction est capitale » (Tardieu 57).

Selon Michelle Perrot, « cette indulgence [pour la femme criminelle] est aussi une façon de la nier » (Perrot 78). C'est aussi exactement ce que dénonce Foucault : « on punit des agressions mais à travers elles, se sont des perversités, des meurtres qui sont aussi des pulsions, des désirs [...] des passions, des instincts, des anomalies, des effets de milieu ou d'hérédité » (Foucault 25).

Edmond de Goncourt traite ou du moins, pense traiter de façon directe, les « conditions d'émergences de la folie» chez le personnage d'Elisa soumise au système carcéral d'Auburn. Cette question est au cœur de l'approche foucaldienne : il s'agit moins de savoir d'où vient le pouvoir, qui le détient et quelles sont les intentions de ceux qui l'ont, que d'étudier les processus par lesquels les sujets sont constitués comme objets du pouvoir. Comment un individu donné, dans un contexte social particulier est-il affecté par les relations de pouvoir en termes d'affirmation de l'identité, des attitudes et prédispositions psychologiques (Layder 101)? C'est précisément ce qu'entend démontrer l'ainé des Goncourt : démontrer que le lien entre le contrôle du corps de la prisonnière et la folie se fait à ce niveau. Dans l'enceinte de la prison, la torture morale s'est substituée à la torture physique - en un système de surveillance impersonnel et envahissant basé sur le panoptique et concentré sur la psychologie de l'individu, ici, en le privant de la parole. Le silence, d'abord mis en place pour obliger la prisonnière à «méditer » sur ses actes, est ensuite imposé pour éliminer toute manifestation de mythomanie et d'hystérie. Exclue de droits civiques, c'est par son corps que la femme s'exprime chez Goncourt. L'irresponsabilité reconnue de la femme criminelle renforce sa passivité en un mouvement de modification de sa personnalité dont le but ultime est le retour au droit et à la vertu (Gelfand 56). Ce faisant, les effets sur la prisonnière sont de la réduire à un être dépersonnalisé, hébété, aux fonctions intellectuelles engourdies. Cette privation se fait si bien « qu'Elisa commença à descendre, peu à peu, tous les échelons de l'humanité qui mènent insensiblement une créature intelligente à l'animalité » (FE 206). Comme le dit Foucault, le «châtiment agit en profondeur dans la pensée, la volonté, la disposition» (Foucault 24). Edmond dépeint la maladie mentale comme la négation de la raison, une régression à l'état de nature : par des indications physiologiques tout d'abord, de par « un endurcissement de son corps que semblait quitter la sensibilité » (FE 185) et un appétit d'une "voracité animale », signe, pour les Goncourt de la déshumanisation, le "prodromeiv de l'imbécilité " (FE 200). C'est donc de « débilitation intellectuelle» (FE 195) qu'il s'agit ici. Par la disparition de conduites supérieures, la folie ferait entrevoir dans le vide creusé par l'absence de fonctions complexes, « la préhistoire de l'humain ». Est décrit ici le phénomène psychologique de la régression provoqué par le «monstre social » qu'est la prison de femmes et qui exclut définitivement l'individu de la raison. Or, d'après Foucault, «la régression ne doit pas être prise comme un des aspects descriptifs de la maladie » (Gros 11).

$\mathrm{Au}$ contraire, la personnalité d'Elisa se réorganise dans la folie selon un style propre qu'il convient de dégager, sa régression n'est alors pas une chute naturelle dans le passé mais une fuite intentionnelle hors du présent (Gros 12-13). Selon l'interprétation foucaldienne, la folie d'Elisa ferait donc partie d'une stratégie de substitution liée à un mécanisme de défense, de repli sur soi. Le refuge dans le passé réveille certaines angoisses liées à son histoire individuelle mais donne aussi une dimension fondamentale à sa propre existence. Là, selon Foucault, dans la folie résiderait la force rebelle de la prisonnière face à la société qui la juge et la condamne. C'est donc à ce point précis de l'angoisse que la folie émergerait. C'est ce que Foucault décrit dans Histoire de la Folie : 
ce qui se trouve au fondement de ces formes pathologiques (névrose, hystérie, folie...), c'est le conflit au sein d'une société, entre les formes d'éducation de l'enfant où elle cache ses rêves et les conditions qu'elle fait aux adultes, où se lisent au contraire son présent réel, ses misères. (Foucault $H F$ 85)

L'expérience subjective conflictuelle, cœur existentiel de la folie, vient trouver dans les contradictions de la société, son fondement secret. «Il y eut comme la rentrée d'une cervelle de petite fille [...] les souvenirs les plus récents, se levaient, s'avançaient des souvenirs anciens, les souvenirs d'une première enfance » $(F E 208)$ qui reprennent possession du corps d'Elisa. C'est dans ses secrets, ses silences, sa vérité intrinsèque, le creux que la folie doit être considérée dans l'œuvre de Goncourt, mais nous y reviendrons plus loin.

Edmond de Goncourt note dans son étude du «cas » d'Elisa que, pendant son enfance, elle avait contracté la fièvre typhoïde deux fois entre 7 et 13 ans. Les effets de cette fièvre sont peu explorés par R. Ricatte qui note simplement dans La Genèse de La fille Elisa que Jules avait écrit en gros caractères dans le carnet f0 8 « effets de la fièvre typhoïde » (Ricatte 59). Il est difficile de trouver, même dans les traités médicaux contemporains, les véritables conséquences à l'âge adulte, de cette maladie contractée pendant l'enfance. Cette fièvre pourrait néanmoins créer une forme dite « sensorielle » de la folie qu'Auguste Voisin, à la fin de son traité, décrit et plus particulièrement, les lésions prodromiques qui la provoquent. Les « lésions de l'innervation cérébrale conduisent toujours à des lésions sympathiques ». " La folie, suite à la fièvre typhoïde, produit une altération méningoencéphalique [...]» (Voisin 108). Elle provoque un épaississement des méninges, des adhérences multiples, un épanchement de sérosité sous-arachnoïdienne v et une hyperémie ${ }_{v i}$ de la substance grise. "L'anémie, la cachexie $_{\text {vii, l'asthénie }}$ viii, les altérations septicémiques du sang, l'élément pestilentiel étaient seuls en cause, aussi, les autopsies ont montré des lésions » (Voisin 121). Voisin conclut que la fièvre typhoïde, chez l'enfant, contribue à l'affaiblissement de l'intelligence, à une imbécilité et idiotie prononcées. Il reste encore à prouver qu'Edmond ait bien lu le traité d'A. Voisin mais il semble plus probable qu'il ait consulté celui d'Alexandre Axenfeld (Goncourt II : 770). D'après Ricatte, comme nous le verrons plus loin, Edmond se serait basé principalement sur les travaux des docteurs Lélut et Béranger. Cependant A. Axenfeld, dans son Traité des Névroses, semble aussi considérer la fièvre typhoïde comme une cause organique indirecte de la folie «agissant en modifiant la composition du sang » (Axenfeld 21), une altération dans l'innervation. L'intelligence est donc atteinte dans son siège par une suite de lésions de la substance corticale ; la parole peut-être perdue ou troublée, lente ou embrouillée. Les dents, le palais, sont également atteints de rétrécissement, pouvant éventuellement neutraliser/troubler la prononciation (Voisin 125). Voisin ne fait pas mention de conséquences ultérieures de la fièvre typhoïde contractée chez l'enfant mis à part une altération de l'intelligence. Axenfeld, quant à lui, explique que la fièvre typhoïde provoquerait des phénomènes hystériques sous la forme de troubles de la sensibilité (Axenfeld 1047). A posteriori, nous pouvons mentionner les travaux de Pierre Janet, qui confirment les hypothèses avancées ci-dessus par Voisin, Axenfeld et Edmond de Goncourt. Dans son traité Névroses et Idées fixes paru en 1898, Janet explique que « la maladie mentale remonte toujours à la petite enfance et dépend de la fièvre typhoïde et des prédispositions particulières du cerveau, notamment, l'hérédité » (Janet 203). Ses prédécesseurs étaient engagés sur la bonne voie et annoncent ainsi l'avènement de la psychanalyse développée avec l'approche freudienne du comportement criminel. En effet, celle-ci prend en considération les émotions de l'individu, son milieu familial durant l'enfance en se basant sur «la parole» du patient, c'est-à-dire, contrairement à Elisa, en lui donnant les capacités physiques à parler de son crime.

Les effets de cette fièvre sont donc susceptibles de se retrouver chez Elisa adulte qui devient prostituée. Cette figure est celle qui exerce une sexualité dite «périphérique » selon Foucault - qui, au 19e siècle, devient un enjeu, une particularité «médicale » et sociale, et qui a son entrée dans les traités médicaux. Comme nous l'avons vu, elle a été intégrée par le discours médical en tant que pathologie, maladie, pour ainsi donner corps à une dichotomie du normal et du pathologique. Selon J.J. Virey, Docteur en médecine à la faculté de Paris « l'abus des voluptés a des effets analogues à ceux de la castration : affaiblissement de l'esprit, impuissance » (Virey 253). La science est alors complice de la construction de cette représentation de la femme, science qui selon Foucault, participe à la production du discours de pouvoir par la connaissance et constitue le pouvoir de la connaissance (Foucault $A S$ 21). Le milieu dans lequel Elisa grandit est construit par les deux frères dans leurs carnets préparatoires. Il s'agit d'un milieu pauvre et insalubre qui « dès le berceau [l'initie] à tout ce que les enfants ignorent de l'amour» ( FE 24). La répulsion d'Elisa pour sa mère, sage-femme, son «caractère intraitable » ( $F E$ 22) d'enfant capricieuse nourrissent en elle la paresse et le défi qui sont les raisons pour lesquelles elle devient prostituée. Elisa est catégorisée comme «monstre biologique », son acte meurtrier est présenté comme prenant sa source dans sa déviance sexuelle. Le personnage s'inscrit dans ce qu'Arlette Farge nomme la théorie de l'" aller et retour » reprise par E Gelfand dans sa partie sur la criminalité féminine, c'est-àdire l'interdépendance des causes et effets entre les situations qui provoquent la criminalité des femmes (instabilité économique, défense de son foyer, enfants non désirés) et les situations qui impliquent une agression envers celles-ci (viol, abandonnement, adultère...) (Gelfand 42). Comme pour Germinie Lacerteux, qui, elle, n'a 
pu se défendre, le viol ou la tentative de viol subie par Elisa est une agression qui exprime en soi la plus extrême violence faite à une femme puisqu'elle est à la fois brutalité physique et torture mentale. Les Goncourt, dans Germinie Lacerteux, connaissent et démontrent de fait que la dimension psychologique du viol ou de l'agression physique est le marquage mental qui contribue à détruire l'individu. Féminité, pauvreté et criminalité sont au cœur du discours social qui repose avant tout sur un discours médical cherchant à préserver «l'ordre social» établi par la bourgeoisie.

La prostituée devient alors l'archétype du corps féminin sexualisé. Alain Corbin décrit la formation et la déformation, durant le $19^{\mathrm{e}}$ siècle, des corps féminins et leur connexion avec le discours social (Corbin 209-19). La prostituée est un corps qui sent mauvais parce que le sang est pourri. D'après Corbin, son corps est immédiatement lié à l'image de l'égout dans lequel le corps social exècre ses excès. Les Goncourt observent lors de leur visite à la prison de femme en 1862 que « le grand signe de la fille tombée à la prostitution, c'est l'impersonnalité [elle devient] l'unité d'un troupeau. Le moi disparait d'elles, c'est-à-dire la conscience et la propriété de soi [...] : elles n'ont plus qu'une âme à la gamelle » (I : 771).

La prostituée est alors symboliquement liée à la mort, à la maladie et plus particulièrement à la syphilis. Elle doit être tolérée dans ce rôle et en même temps surveillée, enfermée, incorporée à un réseau d'observations médicales et de traitements. Dans le roman, il n'est pas fait mention de la syphilis pouvant affecter Elisa mais sa folie pourrait être issue de la maladie vénérienne, alors reconnue comme cause véritablement organique de la folie. La syphilis secondaire peut stimuler une névrose préexistante ou en réveiller d'autres à l'état latent.

Elle n'agit pas directement mais arrive à produire les accidents nerveux par une voie détournée en créant, en accentuant encore un état plus ou moins anémique auquel s'ajoutent les excès, les ennuis, les émotions, les préoccupations, le chagrin. (Axenfeld 1054)

En 1876, le Dr Fournier a accordé un rôle majeur à l'ataxie comme symptôme non négligeable de la folie syphilitique. Mais surtout, il ne faut pas oublier que c'est de cette maladie qu'est mort Jules de Goncourt en 1870, ayant présenté dans son agonie détaillée par Edmond dans le Journal, des symptômes ataxiques. Il ne fait pas de doute que c'est en partie pour cette raison qu'Edmond s'est autant investi dans ce projet littéraire.

Le discours sur la folie est au cœur de cette œuvre même s'il se caractérise par une présence en creux. Ceci est illustré par le rôle du médecin légiste dans les affaires criminelles, qui condamne Elisa, non plus à la peine de mort mais à la prison à perpétuité. Cela montre évidemment les lacunes scientifiques de l'auteur mais ce creux joue aussi du silence qui parcourt le roman où le discours direct est quasi-inexistant. Ce roman porte le sceau du silence représenté par la rareté des dialogues (huit seulement), le recours au style indirect - «elle en avait plein le dos de l'existence avec sa mère... l'ouvrage du bazar était trop abîmant... elle ne voulait pas devenir une tire-enfants... voici bien des semaines qu'elle l'attendait... c'était fini, elle avait pris son parti de donner dans le travers... » (FE 27) - ainsi que les descriptions « expertes » de l'auteur/physiologiste/historien qui envahissent le roman comme ils envahissent l'esprit. Dans la prison, telle une chape de plomb, le silence tombe sur les épaules de la prisonnière avec sept répétitions de la même phrase:

\begin{abstract}
le travail, sans une parole, sans un mot, sans une exclamation [...] Le Silence continu ! C'est tellement contre nature pour une créature humaine de se déshabituer de parler ! [...] Ne jamais parler ! Mais elle était femme, un être dont les sentiments, les sensations, l'impressionnabilité d'enfant, bon gré, mal gré, jaillissent au dehors en une loquacité gazouillante, un verbe diffus, des paroles, beaucoup de paroles. Ne jamais parler! Ne jamais parler ! [...] Ne jamais parler !.... (FE 146)
\end{abstract}

Le processus de déshumanisation mène aux portes de la folie qui d'après Foucault, est exclue de la sphère du langage. C'est dans le silence que la folie s'exprime : la « carence des mots » produit néanmoins une « réserve de sens » (Gros 106-107). C'est par ce silence que la technique de l'aveu, chère à Foucault, est réalisée ici. Encore une fois, c'est un personnage qui s'exprime par le corps avant tout :

[L]a rébellion de son cœur mutiné ne se manifestait par aucun acte, aucune parole, aucune infraction à la discipline : elle était dans son regard, dans son attitude, dans son silence, dans le bouillonnement colère d'un corps terrassé, dans le frémissement d'une bouche qui se tait. (FE 149)

Le silence devient aphasie au moment où « ne pouvant parler, elle ne pouvait résister à se donner le semblant de la parole, à construire avec des lèvres et une langue aphones des phrases qu'elle ne s'entendait pas, mais se sentait dire » (FE 174). Ce verbe en révolte marque la dernière étape d'un esprit encore volontaire jusqu'à ce que l'institution, par le silence, ait raison de sa résistance morale : «Elle se sentait vaincue. Elle s'avouait brisée par la force toute-puissante et toute destructrice de la prison, par la compression de fer qui pesait sur elle » (FE 180). Les prisonnières condamnées au silence développent une forme de solipsisme, plaçant l'individu seul 
devant la seule connaissance de sa propre existence, non pas en tant que transcendance spirituelle puisque son intelligence a été reniée puis détruite, mais en tant qu'enfoncement de la mémoire qui «s'en allait et se perdait parmi les espaces vides » (FE 208).

Ce solipsisme constitue fondamentalement, pour Goncourt, le berceau de la folie car si c'est un « roman à thèse » que voulait écrire Edmond ce n'est pas là que réside la réussite de son roman. Selon Ricatte, ses propos semblent même ne pas suivre ceux des Dr Lélut et Baillarger qu'il a pris comme référence. Or, le Dr Lelut insiste sur le fait que «bien souvent la folie est antérieure à l'emprisonnement et le régime de la prison détecte bien des cas qui, dans la vie courante, auraient échappé aux psychiatres » (Ricatte Romans des Goncourt 39). Malgré lui, c'est bien ce que fait Edmond dans la première partie de son roman : il décrit les prodromes, même les plus imperceptibles pouvant justifier la folie chez Elisa plus tard. Folie qui émerge, Edmond l'écrit luimême, au moment où Elisa tue son amant: «Elisa chez laquelle la courte lutte [avec son amant] avait fait monter au cerveau la folie d'une de ces homicides colères de prostituées» (FE 169). C'est précisément pour cette raison en partie que le roman va dans le sens contraire de ce qu'il est censé démontrer (Michot-Dietrich 323-32). De plus, Goncourt fait mention de la doyenne de la prison qui « avait gardé sa santé et sa raison malgré les trente-six ans passés là » ( $F E$ 130). Ricatte, en disant qu'Edmond aurait même faussé les textes de Baillarger en « remaniant» leur interprétation, ne semble pas saisir que l'enjeu pour Edmond est peut-être moins de faire vrai ici que de transformer l'information scientifique au profit de son propre discours subversif, logé dans le style. Car il y a bien un discours subversif social avec la mise en scène des victimes de la société : c'est la misère et en ce sens, Elisa est bien une représentante du peuple qui est rendue responsable. Mais c'est aussi un discours sur les victimes du pouvoir de l'institution pénitentiaire en tant qu'elle pratique un contrôle moral et psychologique jusqu'à la destruction de l'individu en lui ôtant la parole. Edmond de Goncourt montre enfin ici combien l'absence de parole est destructrice. «Pour la Fille Elisa, me voilà comme un chirurgien qu'on arracherait à un cabinet d'aimables curiosités, obligé de reprendre la cruelle autopsie moderne, la brutale prose, le travail qui fait mal » (II : 704).

La peur d'Edmond de la disparition du mot écrit, de la dégénérescence du style est représentée symboliquement par le geste évocateur de la prisonnière. Celle-ci découpe les lettres de son livre de prière, crée un assemblage, un collage rudimentaire sur du carton où « elle avait formé des mots qu'elle avait collés avec de la mie de pain sur le fond d'une boite à veilleuse » (FE 154), collage destiné à se décomposer. Les mots écrits, et peut-être par extension la littérature, deviennent à leur tour un outil du contre-pouvoir, voire, ici de résistance, en donnant, au travers le silence, la parole, une voix, à ceux qui ne l'ont pas. Enfin, pour finir, cette phrase « Je veux mais je ne peux pas » (FE 198) représente les seuls mots d'Elisa reportés directement, son seul aveu émis « dans le déchirement d'une voix désespérée, $[\ldots]$ dont le corps était agité des pieds à la tête par une trépidation étrange » (FE 198). Uniques paroles véritablement expulsées par un corps en état de souffrance extrême qui sont l'écho direct des derniers mots de Jules en train de mourir en 1870.

Ce roman à thèse finit par échapper à Edmond qui essuie un double échec, il passe à côté de son projet : les législateurs ne l'ont pas entendu et parce qu'il a voulu alerter et émouvoir l'opinion publique, celle-ci s'est simplement limitée à critiquer sa peinture de la prostitution. Ce roman est un témoignage avant tout car il attaque la société sur au moins trois aspects : la pathologie de la folie, la question de l'institution pénitentiaire mais aussi la condition de la femme prostituée. Femme certes primitive à «l'esprit mobile, intentionné, distrait, fuyant, vide et plein de vague, ne pouvant s'arrêter sur rien, incapable de suivre un raisonnement...» (FE 81) mais néanmoins dotée d'une capacité à souffrir et d'une volonté pour se sortir de la condition féminine la plus basse. Au chapitre XXXI, à travers Elisa, Edmond de Goncourt montre clairement que la prostitution n'est pas criminelle mais juste la conséquence d'une condition sociale : « un travail sans beaucoup plus d'attraits que le gagne-pain avec lesquels la pauvreté de la femme conquiert le boire et le manger » (FE 115). La prostitution n'est pas le fait d'une aberration physiologique mais la cause d'une détresse morale - «tête en tourment », « chagrine », « triste », « anxiété », « ennuyée », « disposition hypochondriaque de l'âme blessée » (FE 117) dont les conséquences physiologiques provoquent la souffrance: "envie de pleurer [sans] motifs", « resserrement douloureux du gosier qui lui faisait, une minute, l'effet de se durcir dans le cou », « succession de petites agitations » (FE 116). Enfin, Edmond montre aussi que la femme prostituée n'est pas, comme le discours médical le clame durant tout le $19^{\mathrm{e}}$ siècle, déterminée puisque sa conscience provoquait «une répugnance insurmontable et comme un soulèvement de dégoût et d'horreur pour sa tâche amoureuse à la maison.» (FE 117). Avec La Fille Elisa, Goncourt prend part à la vie de la communauté pour dénoncer l'institution carcérale et la violence, les ravages psychologiques qu'elle peut avoir sur les individus. Premier roman du $19^{\mathrm{e}}$ siècle à traiter la prison - pour la femme - comme un problème et à remettre en cause son pouvoirix, Edmond a voulu démontrer que c'est un lieu créateur de la folie au sens où elle accentue et exacerbe des tensions préexistantes chez l'individu, car la première partie met bien en place les prodromes de la folie appelés à se développer chez Elisa. La prison n'est qu'un catalyseur mais la violence qui s'y exerce assure une descente vers la folie que la vie en société aurait peut-être évitée. La prison est, selon les termes de Foucault, 
une «surface d'émergence » (Foucault $A S$ 17) dans laquelle les relations entre l'autorité de la décision médicale et celle de la décision juridique se cristallisent pour former un pouvoir. Le fait et les formes de l'aliénation mentale sont gouvernés par des pratiques sociales et historiquement situées qui portent les stigmates de la violence ou de l'oppression que la société exerce sur Elisa. De fait, Elisa est trois fois prisonnière, premièrement, de sa condition sociale issue de la misère, ensuite, prisonnière de la maison close et du commerce de son corps et enfin, prisonnière condamnée au silence à qui on dénie le droit élémentaire de l'humain à la parole. Cette violence est caractérisée d'abord, par une atteinte au corps par la tentative de viol, puis, par une intériorisation, un non-dit symptomatique que Goncourt s'efforce de faire remonter à la surface. Il opère une transposition symptomatique du trouble psychologique, non seulement dans le «style-artiste » - écriture qui a pour finalité de réduire au minimum l'écart, le creux, le vide qui existe entre la sensation et la représentation, style qui serait leur propre surface d'émergence - mais également la composition de l'œuvre, caractérisée par le découpage, la discontinuité, la faille. L'œuvre goncourtienne, au-delà des intentions premières de l'auteur, se constituerait donc en obstacle, en objet subversif vis-à-vis d'une société bourgeoise haïe et méprisée parce que créatrice de violence dans sa coercition normative. La femme et sa sexualité surchargée représentent une menace sociale indéniable en ce qu'elle pourrait stimuler une foule populaire et faire monter la violence. Même si cette problématique est plus exploitée chez Zola, en remettant en question le statut criminel et déterminé de la femme prostituée, Edmond de Goncourt annonce les prémices de changements sociaux importants pour les femmes françaises à la fin du $19^{e}$ siècle et remet en question leur statut social. C'est bien plus dans cette subversion inconsciente que l'on peut trouver la vérité de l'œuvre des Goncourt (celle de Jules et Edmond ensemble puis d'Edmond seul) que dans une authentique vérité dans l'engagement au côté du peuple qu'au fond ils méprisent :

Cela est le peuple et je le hais. Dans sa misère, dans ses mains sales [...] je le hais dans tous ses vices tout crus, dans sa prostitution toute nue [...] ! Tout mon moi se soulève contre ces choses qui ne sont pas de mon ordre et contre ces créatures qui ne sont pas de mon sang. (I : 274)

La question sociale est abordée de biais. A travers les circonstances particulières de la protagoniste, se dessine en filigrane « le monde sous le monde » (Préface de Germinie Lacerteux). Cette étude de cas illustre une fois de plus que pour les Goncourt (Edmond continue ici ce qui avait été amorcé à deux) l'enfance est le moment dans lequel les jalons d'un dérèglement particulier sont posés. Cette névrose s'infiltre dans l'individu et se nourrit des attentes déçues, des espoirs sapés, des angoisses créés au contact de l'institution contre laquelle il vient se heurter. La maladie peut alors éclater au grand jour.

Oxford Brookes University

\footnotetext{
Notes

i Sa condamnation repose en fait sur la typologie du crime passionnel sur laquelle nous revenons plus loin dans l'article.

ii Système carcéral de rigueur dans les prisons centrales pour femmes depuis 1839 - importé d'Amérique par Toqueville en 1833, qui oblige les détenues à garder le silence absolu tout en travaillant et vivant en communauté. Un autre système, appelé de Philadelphie qui prône le régime cellulaire est appliqué dans les prisons départementales et ces deux «méthodes » d'incarcération font l'objet d'un débat important en France à cette époque. Modèles punitifs largement décrits par M. Foucault dans Surveiller et Punir, Gallimard, 1975, pp.146-148 et pp. 274-277 mais aussi par Michelle Perrot, «Délinquance et système pénitentiaire en France au XIXe siècle», Annales d'Economie, des Sociétés et des Civilisation, Vol 4, Jan-Fev 1975.

iii Encore une fois, le terme "démence" pose des problèmes puisque selon Tardieu « elle n'est autre chose que l'abolition complète ou incomplète, le plus souvent graduelle des facultés et forme le dernier degré d'un grand nombre d'affections mentales qui aboutissent fatalement à la destruction de l'intelligence. Etat secondaire qui termine plusieurs formes de la folie et consomme la perte de l'intelligence » (33). Les circonstances du diagnostique sont elles aussi très difficiles et c'est certainement pour cela qu'Edmond n'en a pas fait part dans le roman. «L'examen médical a souvent lieu à une époque éloignée de l'action [...] et suppose une grande expérience de la part du médecin car les signes de folie peuvent avoir disparus : folie transitoire. Autre difficulté, la folie souvent ne porte que sur un point limité [...] et surtout, il n'existe pas de type unique de la folie » (46).

iv Un prodrome est le signe avant-coureur qui précède une crise ou une maladie.

v Liquide lymphatique qui se trouve dans la membrane intermédiaire qui recouvre les méninges.
} 
vi Ou POURQUOI OU ? Marianne : l'orthographe est différente : avec ou sans le deuxième H (OU parce que, justement, les deux orthographes existent : Hyperémie OU hyperhémie: présence d'une plus grande quantité de sang dans un organe ou une partie d'organe.

vii Dégradation profonde de l'état général, état des malades en phase terminal (cancer, anorexie, HIV).

viii Fatigue (définitions de www.doctissimo.fr consulté le 12 mars 2007).

ix D'autres romans ont suivi mais c'est la La Fille Elisa qui fut le premier écrit sur la prison en tant qu'institution; A. Belot, Alphonsine (1887) : jeune actrice condamnée aux travaux forces à perpétuité pour un crime qu'elle a commis sous hypnose. Elle subit sa peine à la prison centrale de Clermont d'où elle sera transférée à l'asile Ste Anne grâce au docteur Barillot qui la sait non responsable.

\section{Works Cited}

Axenfeld, Alexandre. Traité des névroses. Paris : Baillière, 1883 ( $2^{\mathrm{e}}$ édition).

Corbin, A. Histoire du corps. Vol.2, Paris: Seuil, 2005.

Foucault, Michel. Surveiller et punir. Paris : Gallimard, 1975.

---. Histoire de la folie à l'âge classique. Paris : Gallimard, 1972.

---. Archéologie du savoir. Paris : Gallimard, 1969.

Gelfand, E. Imagination in Confinement. Ithaca \& London : Cornell UP, 1983.

Goncourt, Edmond \& Jule de. Le Journal de la vie littéraire. Paris : Robert Laffont, 1953.

---. La fille Elisa. 1877. Paris : Flammarion, 1956.

Gros, Frédéric. Foucault et la folie. Paris : PUF, 1997.

Harris, R. Murders and madness: legal psychiatry and criminal anthropology in Paris, 1880-1910. D. Phil Thesis, U of Oxford, 1985.

Layder, Derek. Understanding Social Theory. London : SAGE Publications, 1994.

Perrot, Michelle. «Délinquance et système pénitentiaire en France au XIXe siècle ». Annales d'Economie, des Sociétés et des Civilisation. Vol 4, Jan-Fev. $1975: 67-91$.

Perrot, Michelle \& Fraisse, Geneviève. Histoire des femmes en Occident. Vol IV, Paris : Plon, 2002.

Ricatte, Robert. La génèse de La fille Elisa. Paris : PUF, 1960.

---. «Les romans des Goncourt et la médecine ». Revue des sciences humaines (janvier-mars 1953) : 27-43.

Smart, Carol. Law, Crime \& Sexuality Thousand Oaks. Calif ; London : Sage Publications, 1995.

Tardieu, Alexandre. Etude médico-légale sur la folie. Paris : Baillière \& Fils, 1872.

Voisin, Auguste. Leçons cliniques sur les maladies mentales et sur les maladies nerveuses à la Salpêtrière. Paris : Baillière, 1883. 